\title{
PedsQL-4 Emotional Functioning Scale
}

National Cancer Institute

\section{Source}

National Cancer Institute. PedsQL-4 Emotional Functioning Scale. NCI Thesaurus. Code C121526.

A rating scale included in the Pediatric Quality of Life Inventory Version 4 (PedsQL 4.0) that measures problems with a child's feelings and emotions. 